\title{
Transient Ischemic Attack and Carotid Web
}

\author{
(D) H. Hu, (DX. Zhang, (1)J. Zhao, (D) Y. Li, and DY. Zhao
}

\begin{abstract}
BACKGROUND AND PURPOSE: Carotid web was recognized as a cause of ischemic stroke. We sought to determine the clinical and imaging profiles of patients with a carotid web as well as its association with TIA.
\end{abstract}

MATERIALS AND METHODS: A retrospective review of carotid CT angiography studies and brain MR imaging in patients with TIA during the past 3 years $(n=135$ ) was performed to determine the presence of carotid webs by 2 experienced neuroradiologists according to previously published criteria. Demographics and clinical and imaging characteristics are shown by descriptive statistics for patients with an identified carotid web. The agreement in the detection of carotid webs between 2 neuroradiologists was examined using $\kappa$ statistics.

RESULTS: There were 12 (8.9\%) carotid webs at the symptomatic bifurcation and 1 carotid web (0.7\%) at the asymptomatic bifurcation, and no hyperintensity was seen on DWI of these 12 patients. Eight of these 12 (75\%) patients with a carotid web were women. None of the 12 patients with a carotid web had major risk factors or other causes of TIA. Fair-to-good interobserver agreement $(\kappa=$ 0.87 ) was seen for diagnosing a carotid web with CT angiography. The rate (10/12, 83.3\%) of short-term recurrent episodes of TIA in patients with TIA with a carotid web was significantly higher than that of patients without a carotid web $(15 / 123,12.2 \%)(P<.001)$.

CONCLUSIONS: The incidence of carotid web in patients with TIA was $8.9 \%$. There is an association between carotid web and patients with TIA without other identified risks. Carotid web may be an underestimated risk factor for TIA.

$\mathbf{T}$ ransient ischemic attack is a common ischemic cerebrovascular disease. According to the guidelines proposed by the American Heart Association and American Stroke Association in $2009,{ }^{1}$ TIA is defined as a transient episode of neurologic dysfunction caused by focal brain, spinal cord, or retinal ischemia, without acute infarction. The probability of stroke occurring at 2, 7 , 30 , and 90 days after TIA onset was approximately $2 \%-4 \%, 6 \%$,

Received August 25, 2018; accepted after revision December 3.

From the Institute of Diagnostic and Interventional Radiology (H.H., X.Z., J.Z., Y.L.), and Department of Neurology (Y.Z.), Shanghai Jiao Tong University Affiliated Sixth People's Hospital, Shanghai, People's Republic of China.

This work was supported by National Natural Science Foundation of China No. 81871329; National Natural Science Foundation of China No. 81471656; Shanghai Talent Development Fund, No. 201555; Shanghai Key Discipline of Medical Imaging grant No. 2017ZZ02005; Shanghai Municipal Education Commission-Gaofeng Clinical Medicine Grant Support No. 2016427; Clinical Science and Technology Innovation Project of Shanghai Shen Kang Hospital Development Center No. SHDC22015038; and Shanghai Municipal Science and Technology Commission Medical Guide Project No. 16411968900.

Please address correspondence to Yuehua Li, MD, Institute of Diagnostic and Interventional Radiology, Shanghai Jiao Tong University Affiliated Sixth People's Hospital, 600, Yishan Rd, Shanghai, 200233, China; e-mail: liyuehua312@163.com

-- Indicates open access to non-subscribers at www.ajnr.org

http://dx.doi.org/10.3174/ajnr.A5946

$4 \%-10 \%$, and $10 \%-20 \%$ in previous studies. ${ }^{2,3}$ If the patient is treated properly, the risk of stroke at 90 days after TIA could be reduced to $1 \%-3 \%{ }^{4}$ Therefore, TIA is a stroke-warning event requiring urgent intervention, and its occurrence is also a good time for secondary prevention.

Decisions about TIA treatment and secondary prevention rely on identification of the main cause of the disease. However, in one-third of patients with TIA, the cause cannot be identified. ${ }^{5}$ In several recent case series, carotid webs were identified as a possible cause of ischemic stroke in a predominantly female population with no otherwise known reason for stroke. ${ }^{6-9} \mathrm{~A}$ carotid web is defined as a thin intraluminal filling defect along the posterior wall of the carotid bulb in oblique sagittal reformats, and, most important, with a septum evident on the axial section on CTA. ${ }^{6-9}$ However, there are few reports on the relationship between a carotid web and TIA. Is carotid web one of the causes of TIA?

Therefore, we hypothesized that a carotid web may be one of the risk factors for TIA without identifiable cause. We retrospectively analyzed carotid CTA data to explore the incidence of carotid webs in patients with TIA and to determine the clinical and imaging profiles of patients with carotid webs as well as their association with TIA. 


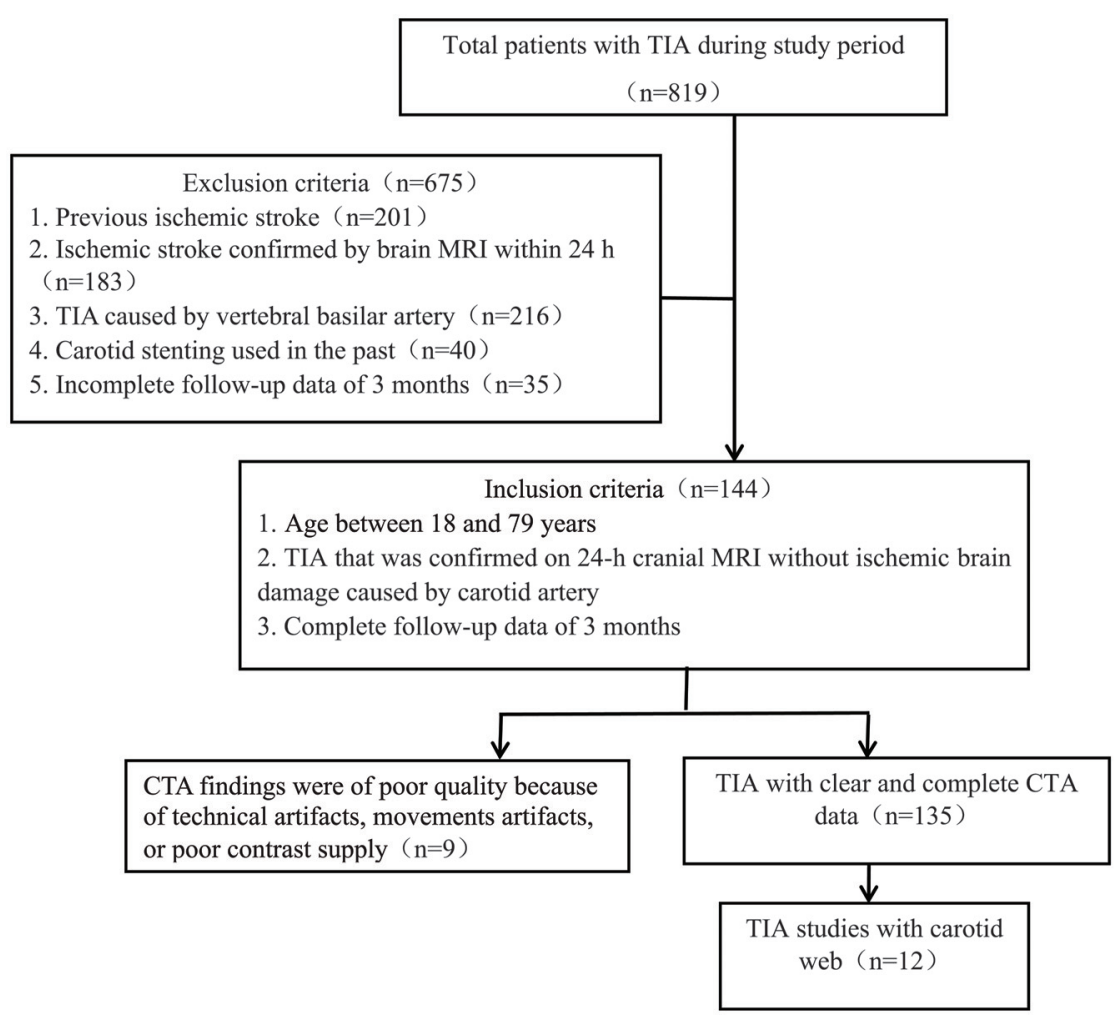

FIG 1. Flow chart of inclusion criteria.
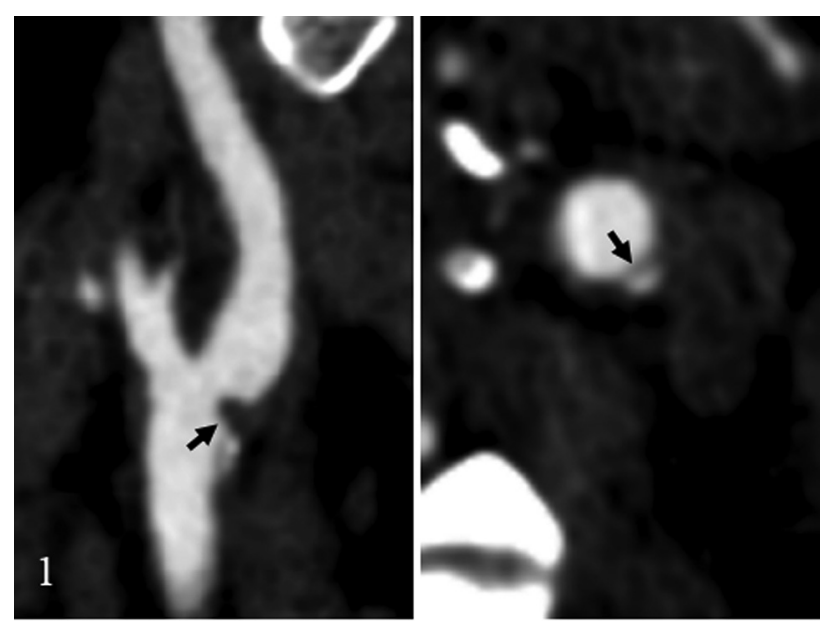

FIG 2. Carotid web diagnosis by the classic appearance on CTA, which shows a thin intraluminal filling defect along the posterior wall of the carotid bulb on an oblique sagittal section and a corresponding septum on axial images (arrow).

\section{MATERIALS AND METHODS \\ Patients}

This retrospective study was approved by the ethics committee of the Sixth People's Hospital of Shanghai Jiao Tong University; the need for informed consent was waived. A chart review of all patients $(n=819)$ presenting to our hospital with TIA from January 1,2015 , to December 31, 2017, was undertaken. Figure 1 provides details of patient selection for the study.

\section{Clinical Information}

Data on age, sex, history of hypertension, diabetes mellitus, cardiac insufficiency, atrial fibrillation, and current smoking were retrospectively collected. All patients were treated with antiplatelet therapy. Data on routine follow-up within 3 months after discharge were also collected. A short-term recurrent episode was considered if TIA relapse occurred during the first hospitalization or within 3 months, and short-term progression of acute cerebral infarction was considered if an ischemic stroke was confirmed on MR imaging.

\section{Image Evaluation}

Carotid CTA was performed on a 128 multidetector row CT scanner with 1-mm thick sections and was acquired from the aortic arch through the circle of Willis. Scanning parameters included the following: gantry rotation time, 0.5 seconds; pitch, 1; voltage, $120 \mathrm{kV}$ (peak); and current, $360 \mathrm{mAs}$. Intravenous contrast material $(80 \mathrm{~mL}$ of iohexol, 300 $\mathrm{mg} / \mathrm{mL}$, Omnipaque; GE Healthcare, Piscataway, New Jersey) and a $50-\mathrm{mL}$ saline flush were administered at a rate of $4 \mathrm{~mL} / \mathrm{s}$ with a power injector. Images were reconstructed with axial, coronal, sagittal, and 3D volume reconstructions.

An independent review of these studies was then performed by 2 experienced neuroradiologists with 6-8 years of experience in carotid CTA imaging and analysis. The readers were blinded to patient age and sex. The presence of a carotid web was evaluated by each reader at the baseline CTA. A carotid web was identified when there was a thin, smooth, membrane-like intraluminal filling defect along the posterior wall of the carotid bulb on oblique sagittal images and a corresponding thin septum on axial images (Fig 2). ${ }^{5-8}$ Then, there was a consensus reading of all webs identified by the 2 radiologists independently. A carotid web was diagnosed only if both radiologists agreed regarding the presence of a web on sagittal and axial images. The degree of carotid stenosis and the length of the carotid web were measured according to NASCET criteria ${ }^{10}$ and the study of Haussen et al. ${ }^{11}$ The differential diagnosis was performed, as shown in Fig 3, when calcified atherosclerotic plaques, carotid artery embolism, carotid artery dissection, or typical fibromuscular dysplasia at $3 \mathrm{~cm}$ above and below the carotid bifurcation was detected. FLAIR and DWI imaging were used to assess the presence of TIA-induced brain tissue damage. Follow-up brain MR imaging (DWI and FLAIR) was assessed for the presence of stroke.

\section{Statistical Analysis}

Demographic data and clinical information were presented as medians. For patients both with and without a carotid web on CTA, group differences in age were assessed using the MannWhitney $U$ test. For other baseline values (demographics and risk factors), group differences were assessed using the Fisher exact test. The proportion of carotid webs at the symptomatic and asymptomatic bifurcations was estimated using conditional logis- 

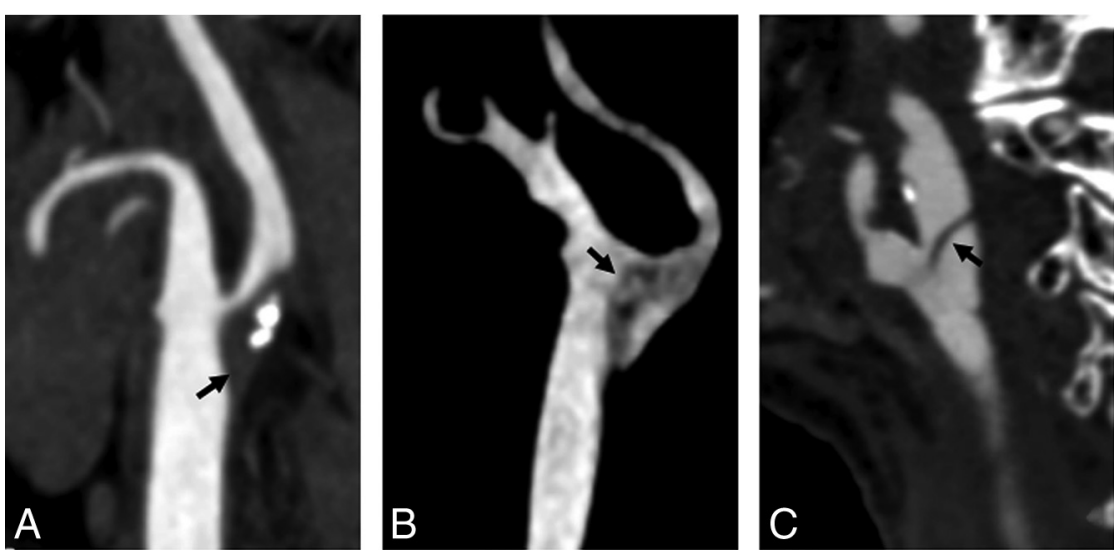

FIG 3. The differential diagnosis includes calcified atherosclerotic plaques $(A)$, carotid artery embolism (B), and carotid artery dissection (C).

tic regression, in which odds ratios were calculated with $95 \%$ confidence intervals. The Cohen $\kappa$ statistic was used to calculate the interobserver agreement for identifying carotid webs for all patients. Statistical significance was defined as $P<.05$. Analyses were performed with SPSS 22.0 (IBM, Armonk, New York).

\section{RESULTS}

Of the 135 patients with TIA with complete carotid CTA data included in this study, both readers agreed on 12 cases of carotid web (Cohen coefficient, 0.87; 95\% CI, 0.55-1). Disagreement was encountered in 2 patients. Furthermore, the 2 cases were both rejected as webs because the 2 readers could not reach an agreement. Radiologically, the typical appearance of a carotid web on CTA is a thin intraluminal filling defect arising from the posterior wall of the carotid bulb on oblique sagittal images and a corresponding thin septum on axial images (Fig 4). There were 12 (8.9\%; 95\% CI, 4\%-13.8\%) carotid webs at the symptomatic bifurcation and $1(0.7 \% ; 95 \% \mathrm{CI}, 0 \%-2.2 \%)$ at the asymptomatic bifurcation (odds ratio, 3.4; 95\% CI, 1.9-6.26; $P<.001$ ). As shown in Table 1 , the median age (58 years) of patients with a carotid web at the symptomatic carotid bifurcation differed $(P=$ .026) from those (64 years) without a carotid web at the symptomatic carotid bifurcation. We found that carotid webs in the symptomatic carotid artery were more often observed in female patients $(n=9)$ than in male patients $(n=3 ; P<.015)$. However, there were no differences in symptomatic carotid artery location between the right and left sides $(P=.548)$. Among 12 patients with a carotid web at the symptomatic side, 1 had a history of smoking (case 2), one had a history of diabetes (case 3), and 10 had no risk factors for TIA such as hypertension, diabetes, atrial fibrillation, and myocardial infarction.

All 135 patients with TIA underwent brain MR imaging within 24 hours after admission. Carotid CTA was performed within 1 week. There was no hyperintensity detected on DWI, in both patients with symptomatic carotid webs and those without carotid webs. The rate $(10 / 12,83.3 \%)$ of short-term recurrent episodes of TIA in patients with TIA with a carotid artery web was significantly higher than that of patients without a carotid artery web $(15 / 123,12.2 \%)(P<.00)$.

As shown in Table 2, the degree of carotid artery stenosis (NASCET criteria) ${ }^{10}$ was $<50 \%$ (range, $0 \%-20 \%$ ), and the aver-

\section{DISCUSSION} found. populations. . $^{8,13}$ age length of the carotid web was $3.9 \pm$ $1.1 \mathrm{~mm}$ (range, $2.9-6.5 \mathrm{~mm}$ ) in all 12 patients with carotid webs. Furthermore, the average size $(3.5 \pm 0.8 \mathrm{~mm})$ of the carotid artery web in patients with a carotid web without recurrent TIA and progressive ischemic stroke within 3 months was smaller than that $(4.1 \pm 1.2$ $\mathrm{mm}$ ) in patients with carotid web with recurrent TIA and deteriorating ischemic stroke within 3 months $(P=$ .416). All 12 patients with a carotid web had no typical medial fibromuscular dysplasia. Three of 12 patients had nonstenotic calcification at the carotid bifurcation.

Our findings showed a prevalence of $8.9 \%$ of symptomatic carotid webs at the carotid bifurcation in patients with TIA due to carotid system disease. Other notable major risk factors for TIA could not be identified in most patients with a symptomatic carotid web. These findings support our hypothesis that a carotid web may be an important risk factor for ischemic TIA when no other cause is

We found a higher prevalence $(8.9 \%)$ of carotid webs in patients with TIA than in previously reported data $\left(1.2 \%{ }^{6}\right.$ and $2.5 \%{ }^{9}$ ) according to a hospital-based sample of patients suspected of having stroke and intracranial large-vessel occlusion, respectively. TIA, a precursor of ischemic stroke, carries a high risk of recurrent stroke within 90 days from the event, ${ }^{12}$ which could explain the higher prevalence. In addition, the previously reported prevalence of a carotid web was $9.4 \%, 21.2 \%$, and $37 \%$, respectively, in patients with cryptogenic ischemic stroke $e^{7,8,13}$ and $23 \%{ }^{13}$ in all patients with carotid ischemic stroke. Our result was similar to the earlier reported prevalence $(9.4 \%)$ of carotid webs in patients with cryptogenic ischemic stroke, but lower than the prevalence of $21.2 \%, 37 \%$, and $23 \%$ in patients with cryptogenic ischemic stroke and all patients with carotid ischemic stroke, respectively. Differences in methodology and population characteristics may explain discrepancies in these findings because the prevalence of a carotid web is thought to be increased in African American and Afro-Caribbean compared with white and Asian

In our data, 2 patients with TIA and a carotid web each had a history of smoking and diabetes. While smoking and diabetes were independent risk factors for TIA, ${ }^{14-16}$ no atheromatous changes were seen on CTA. Thus, a carotid web was the main identifiable cause of our patients' TIAs. Existing literature about the relation of carotid web and TIA is scarce. Morgenlander and Goldstein ${ }^{17}$ reported that a young female patient with a carotid web but without other identifiable risk factors for stroke progressed to acute ischemic stroke after a recurrent TIA. Thus, our findings are in line with those in the literature.

In our study, carotid webs were more frequently found at the ipsilateral symptomatic bifurcation and in female patients without cardiovascular risk factors or other major risk factors for TIA. 

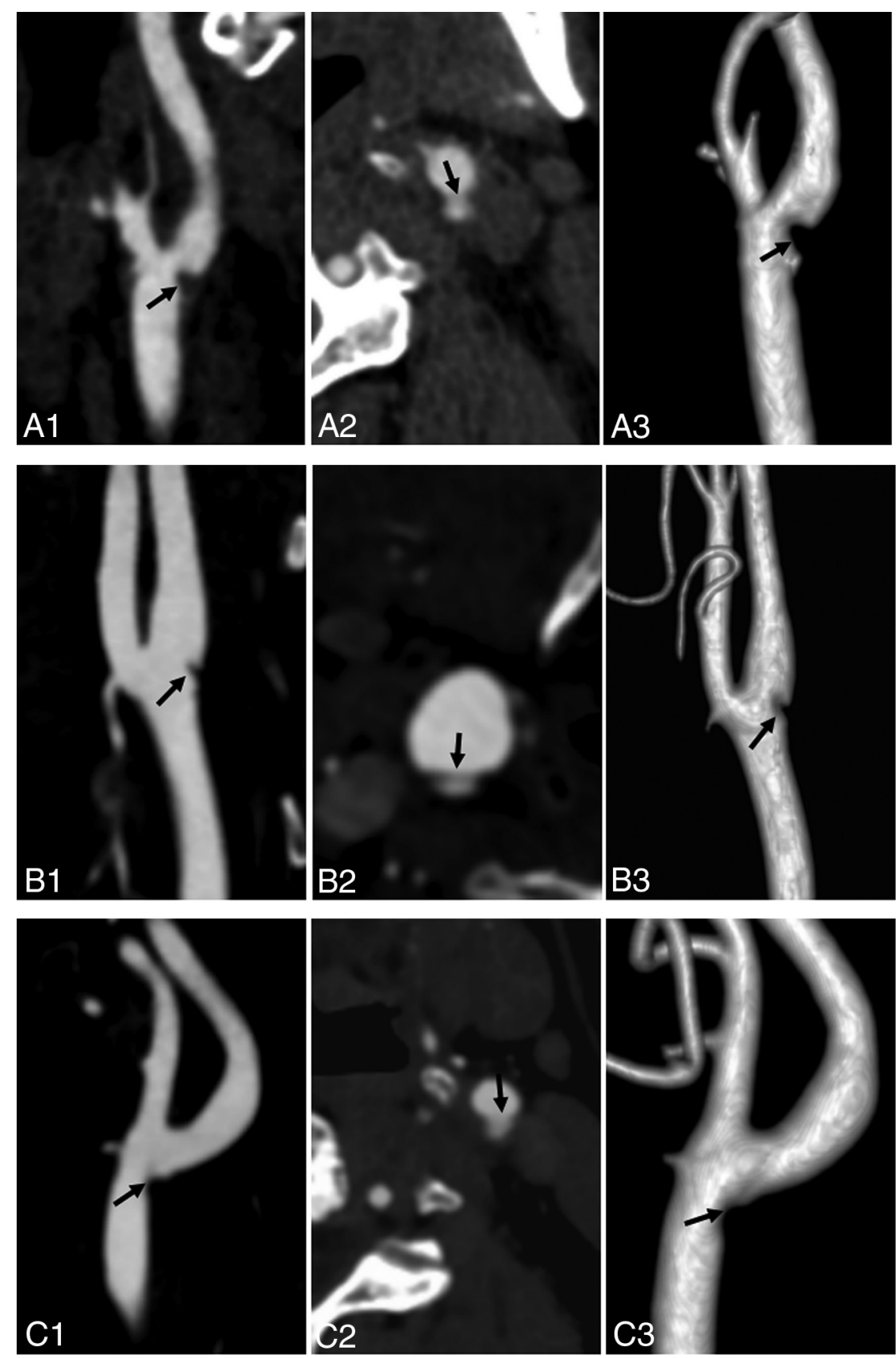

FIG 4. Carotid artery web in 3 patients with TIA $(A-C)$. Sagittal oblique CTA $(A I, B]$, and $C l)$ shows a shelf-like intraluminal protrusion arising from the posterior wall of the carotid bulb (arrow). Axial CTA $(A 2, B 2$, and $C 2)$ shows that the membrane is shelf-like, consistent with a carotid artery web (arrow); volume reconstruction $(A 3, B 3$, and $C 3$ ) shows the same web inside the vessel (arrow).

This result was consistent with literature reports about patients with stroke with carotid webs. ${ }^{6-9,13}$ However, the median age (58 years) of patients with TIA with a carotid web in our study was higher than that previously reported for patients with stroke with a carotid web, ${ }^{6-9,13}$ which may be related to an older age of onset in patients with TIA.

In this study, the rate of short-term recurrent episodes of TIA within 3 months in patients with TIA with carotid webs was $83.3 \%$, higher than that of patients with TIA without a carotid artery web $(12.2 \%)(P<.001)$ and higher than the average reported in the literature. ${ }^{2,3}$ Moreover, the degree of carotid artery stenosis (NASCET criteria) ${ }^{10}$ of all 12 patients with carotid webs was $<50 \%$ (range, 0\%-20\%). Generally, the degree of carotid artery stenosis $<50 \%$ is not considered a risk factor for TIA or ischemic stroke. ${ }^{7}$ Thus, studies have postulated that carotid web-induced ischemic stroke may be mediated by an embolic mechanism, with sluggish/turbulent blood flow produced by the filling defect increasing the thrombogenicity and risk of artery-to-artery embolism. ${ }^{6-9,13,18}$ Therefore, we speculated that the presence of a carotid web might lead to changes of carotid hemodynamics, which was related to recurrence of TIA and progression to acute ischemic stroke within 3 months in patients with TIA with carotid webs.

However, because of the limitation of small samples and lack of fluid dynamics data, the relation between the thrombogenicity of webs and the size of the "shelf" of the web was not clear in this study. Although the average size of a carotid web $(4.1 \pm 1.2 \mathrm{~mm})$ in patients with recurrence of TIA and progression to stroke within 3 months was larger than that $(3.5 \pm 0.8 \mathrm{~mm})$ in patients without recurrence of TIA and progression to stroke within 3 months, there were no statistically significant differences between the 2 groups $(P=.416)$. Hence, the relation between the change of carotid hemodynamics and the size of the shelf of the carotid web in patients with TIA deserves further study.

In our study, the agreement between the 2 neuroradiologists on the CTAbased diagnosis of carotid webs was $\operatorname{good}(\kappa=0.87)$. The carotid web had characteristics on CTA, and it can be definitely diagnosed. Carotid webs could be distinguished from dissection and atherosclerotic plaques by their characteristic location and appearance, being thin and focal in nature and exclusively located in the posterior wall of the carotid bulb. In contrast, dissection is typically located beyond the carotid bulb and may be related to intramural hematomas and/or pseudoaneurysms. Soft carotid plaques often, but not necessarily, are seen as focal areas of vessel wall thickening, confined to the posterior wall of the carotid bulb. ${ }^{9}$ Histopathologically, carotid web has been referred to as an atypical variant of fibromuscular dysplasia, though without actual evidence of fibromuscular dysplasia in other vessels, which is different from the typical presentation of fibromuscular dysplasia. ${ }^{6,13}$ Typical myofibroblastic dysplasia mainly involves the arterial media, 
Table 1: Demographics and clinical characteristics of patients in our study

\begin{tabular}{|c|c|c|c|}
\hline Parameter & $\begin{array}{l}\text { Patients with TIA } \\
\text { without Carotid Web } \\
\text { ( } n=123)\end{array}$ & $\begin{array}{l}\text { Patients with TIA } \\
\text { with Carotid Web } \\
\qquad(n=12)\end{array}$ & $P$ Value \\
\hline Median age (yr) & 64 & 58 & .028 \\
\hline Sex (male) & $77(62.6 \%)$ & $3(25 \%)$ & .015 \\
\hline \multicolumn{4}{|l|}{ Ethnicity (No.) (\%) } \\
\hline Chinese & $123(100 \%)$ & $12(100 \%)$ & \\
\hline Other & 0 & 0 & \\
\hline Right-hemisphere stroke & $56(45.5 \%)$ & $4(33.3 \%)$ & .548 \\
\hline \multicolumn{4}{|l|}{ Risk factors } \\
\hline Hypertension & $26(21.1 \%)$ & 0 & .122 \\
\hline Diabetes & $16(13.0 \%)$ & $1(8.3 \%)$ & 1.0 \\
\hline Atrial fibrillation & $9(7.3 \%)$ & 0 & 1.0 \\
\hline Myocardial infarction & $2(1.6 \%)$ & 0 & 1.0 \\
\hline Smoking & $20(16.3 \%)$ & $1(8.3 \%)$ & .691 \\
\hline Alcohol abuse & $10(8.1 \%)$ & 0 & .599 \\
\hline MRI (DWI hyperintensity) & 0 & 0 & \\
\hline Recurrent episode within $3 \mathrm{mo}$ & $15(12.2 \%)$ & $10(83.3 \%)$ & .00 \\
\hline
\end{tabular}

Note:-Unless otherwise indicated, data are absolute values with percentages in parentheses.

Table 2: Detailed imaging/clinical features of 12 patients with TIA with a carotid web

\begin{tabular}{lcccc}
\hline Patient No. & $\begin{array}{c}\text { Degree of Carotid } \\
\text { Artery Stenosis (\%) } \\
\text { and Carotid Web (mm) }\end{array}$ & $\begin{array}{c}\text { Nonstenosis Calcification } \\
\text { of Carotid Bifurcation }\end{array}$ & $\begin{array}{c}\text { Pecurrent } \\
\text { TIA }\end{array}$ & $\begin{array}{c}\text { Progression to } \\
\text { Cerebral Infarction } \\
\text { (within 3 mo) }\end{array}$ \\
\hline 1 & $0,3.5$ & No & Yes & No \\
2 & $20,6.5$ & Yes & Yes & Yes \\
3 & $0,3.5$ & No & Yes & Yes \\
4 & $10,3.0$ & No & Yes & No \\
5 & $0,2.9$ & No & No & Yes \\
6 & $10,5.8$ & Yes & Yes & Yes \\
7 & $5,3.6$ & No & Yes & Yes \\
8 & $0,3.3$ & No & Yes & Yes \\
9 & $15,3.9$ & No & Yes & No \\
10 & $0,3.0$ & Yes & Yes & Yes \\
11 & $0,4.6$ & No & No & Yes \\
\hline
\end{tabular}

with a classic "string of beads" imaging appearance, and is not associated with ischemic stroke. ${ }^{6-9,13}$

Proper detection of carotid webs could help optimize the management of TIA and stroke; in particular, for patients with TIA and acute ischemic stroke without other clear risk factors. Several treatment options have been proposed, such as carotid endarterectomy and stent placement. ${ }^{10,11,19,20}$ However, optimal treatment strategies need to be investigated in clinical trials.

Our research had several limitations. First, the study design was a retrospective analysis with a small sample size. Second, all patients with carotid webs were identified on the basis of carotid CTA, without histologic observation. Third, although small protruding lesions of a carotid bifurcation were considered a risk factor in previous studies, ${ }^{6,9}$ they were not included in our study because of lower interobserver agreement of assessment during carotid CTA.

\section{CONCLUSIONS}

Our study showed that the incidence of a carotid web in patients with TIA was $8.9 \%$ and the rate of progression to ischemic stroke in the short term was high. Therefore, carotid webs should be considered in patients with TIA who do not have other major risk factors.

\section{REFERENCES}

1. Easton JD, Saver JL, Albers GW, et al; American Heart Association, American Stroke Association Stroke Council, Council on Cardiovascular Surgery and Anesthesia, Council on Cardiovascular Radiology and Intervention, Council on Cardiovascular Nursing, Interdisciplinary Council on Peripheral Vascular Disease. Definition and evaluation of transient ischemic attack: a scientific statement for healthcare professionals from the American Heart Association/American Stroke Association Stroke Council; Council on Cardiovascular Surgery and Anesthesia; Council on Cardiovascular Radiology and Intervention; Council on Cardiovascular Nursing; and the Interdisciplinary Council on Peripheral Vascular Disease-The American Academy of Neurology affirms the value of this statement as an educational tool for neurologists. Stroke 2009;40:2276-93 CrossRef Medline

2. Wu CM, McLaughlin K, Lorenzetti DL, et al. Early risk of stroke after transient ischemic attack: a systematic review and meta-analysis. Arch Intern Med 2007;167:2417-22 CrossRef Medline

3. Giles MF, Rothwell PM. Risk of stroke early after transient ischaemic attack: a systematic review and meta-analysis. Lancet Neurol 2007;6:1063-72 CrossRef Medline

4. Sanders LM, Srikanth VK, Jolley DJ, et al. Monash transient ischemic attack triaging treatment: safety of a transient ischemic attack mechanism-based outpatient model of care. Stroke 2012;43: 2936-41 CrossRef Medline

5. Li L, Yiin GS, Geraghty OC, et al. Incidence, outcome, risk factors, and long-term prognosis of cryptogenic transient ischaemic attack 
and ischaemic stroke: a population-based study. Lancet Neurol 2015;14:903-13 CrossRef Medline

6. Choi PM, Singh D, Trivedi A, et al. Carotid webs and recurrent ischemic strokes in the era of CT angiography. AJNR Am J Neuroradiol 2015;36:2134-39 CrossRef Medline

7. Coutinho JM, Derkatch S, Potvin AR, et al. Carotid artery web and ischemic stroke: a case-control study. Neurology 2017;88:65-69 CrossRef Medline

8. Sajedi PI, Gonzalez JN. Carotid bulb webs as a cause of "cryptogenic" ischemic stroke. AJNR Am J Neuroradiol 2017;38:1399-404 CrossRef Medline

9. Compagne KCJ, van Es AC, Berkhemer OA, et al; MR CLEAN Trial Investigators. Prevalence of carotid web in patients with acute intracranial stroke due to intracranial large vessel occlusion. Radiology 2018;286:1000-07 CrossRef Medline

10. Barnett HJ, Taylor DW, Eliasziw M, et al. Benefit of carotid endarterectomy in patients with symptomatic moderate or severe stenosis: North American Symptomatic Carotid Endarterectomy Trial Collaborators. N Engl J Med 1998;339:1415-25 CrossRef Medline

11. Haussen DC, Grossberg JA, Bouslama M, et al. Carotid web (intimal fibromuscular dysplasia) has high stroke recurrence risk and is amenable to stenting. Stroke 2017;48:3134-37 CrossRef Medline

12. Cruz-Flores $S$. Acute stroke and transient ischemic attack in the outpatient clinic. Med Clin North Am 2017;101:479-94 CrossRef Medline

13. Joux J, Boulanger M, Jeannin S, et al. Association between carotid bulb diaphragm and ischemic stroke in young Afro-Caribbean patients: a population-based case-control study. Stroke 2016;47: 2641-44 CrossRef Medline

14. Wong SW, McGrath NN. Screening, prevalence and ethnic variation of diabetes mellitus in people with acute stroke and transient ischaemic attack: a cross-sectional study in Northland, New Zealand. N Z Med J 2016;129:62-66 Medline

15. Daubail B, Durier J, Jacquin A, et al. Factors associated with early recurrence at the first evaluation of patients with transient ischemic attack. J Clin Neurosci 2014;21:1940-44 CrossRef Medline

16. Strom JO, Tavosian A, Appelros P. Cardiovascular risk factors and TIA characteristics in 19,872 Swedish TIA patients. Acta Neurol Scand 2016;134:427-33 CrossRef Medline

17. Morgenlander JC, Goldstein LB. Recurrent transient ischemic attacks and stroke in association with an internal carotid artery web. Stroke 1991;22:94-98 CrossRef Medline

18. Kim SJ, Nogueira RG, Haussen DC. Current understanding and gaps in research of carotid webs in ischemic strokes: a review. JAMA Neurol 2018 Nov 5. [Epub ahead of print] CrossRef Medline

19. Lenck S, Labeyrie MA, Saint-Maurice JP, et al. Diaphragms of the carotid and vertebral arteries: an under-diagnosed cause of ischaemic stroke. Eur J Neurol 2014;21:586-93 CrossRef Medline

20. Elmokadem AH, Ansari SA, Sangha R, et al. Neurointerventional management of carotid webs associated with recurrent and acute cerebral ischemic syndromes. Interv Neuroradiol 2016;22:432-37 CrossRef Medline 